\title{
The Research on the County's Development State of Cultural Industry in Guizhou Province
}

\author{
Chang-bing YANG ${ }^{1,2,3, a}$ and Mu ZHANG ${ }^{1, b, *}$ \\ 1School of Finance, Guizhou University of Finance and Economics, Guiyang Guizhou 550025, China \\ 2Guizhou Institute for Urban Economics and Development, Guizhou University of Finance and Economics, Guiyang \\ Guizhou 550025, China \\ 3Guizhou Institution for Technology Innovation \& Entrepreneurship Investment, Guizhou University of Finance and \\ Economics, Guiyang Guizhou 550025, China \\ aemail:1532433977@qq.com, bemail:rim_007@163.com \\ *Corresponding author
}

\begin{abstract}
In order to learn of the development state of cultural industry in Guizhou Province, this paper applies principal component analysis with the help of SPSS 19.0.Firstly, we established index system of the development level of cultural industry. Then, we used SPSS 19.0 to standardize the statistics, followingly, we did principal component analysis with the standardized the statistics and we got six principal components. Finally, we applied SPSS 19.0 to analyse the result and got the scores of every principal component, then we used each principal component's eigenvalue as weight to calcuate each county's weighted scores and got the rank based on the weighted scores.
\end{abstract}

Keywords-Guizhou Province; cultural industry; evaluation index; principal component analysis

\section{INTRODUCTION}

It is well-acknowledged that cultural industry is one of the most uprising industries. To develop culture industry is a extremely strategic choice, it is not only beneficial to upgrade industrial structure ,but it is also beneficial to improve each county's well-being[1].In 2015,the added value of culture and its related industries is 2723.5 billion yuan, which increased by 11 per cent compared with last year and this growth rate is 4.6 per cent higher than nominal GDP growth rate.In addition, the added value of culture and its related industries accounts for 3.97 per cent ,which is 0.16 per cent higher than last year. Cultural industry is a bright spot of economic growth and it plays an increasingly important role in economic growth , the scale of gross output of culture industry continuously expands and its proportion in GDP rises fastly. Guizhou is a typical province where lives so many minorities and has obvious national characteristics, therefore, it is even a natural national culture museum. Recently, under the support of our country's policies, Guizhou province has sought to establish the Ten Cultural Industrial Park and the Ten Cultural Industry Base in the whole province and most of them have been established and come into service. According to the statistics, the Guizhou province's gross production value of national artworks and related handicraft article in culture industry has been over 50 billion in 2014 and number of enterprises has reached 2400, which has decreased about 2 million of the unemployment directly and indirectly. During the 13th Five Year Plan, the local government of Guizhou province is actively seeking to develop culture industry and they plan to carry out four key projects including altogether forty four subprojects and the four key projects can be divided into four categories, respectively Park Base, Block and Town, Cultural Industry Project, Continued Project. Although these series actions will bring various and enough benefits to develop local culture industry, Guizhou province is a underdeveloped area and has many constrains on the development of culture industry, such as traffic inconvenience, low per capita income. In view of these facts, Guizhou province people's political consultative conference conduct a series surveys on 88 counties within its jurisdiction, in order to find out each county's disadvantages and shortcomings in the process of the development of culture industry meanwhile to make up these disadvantages and shortcomings so that accelerate the development of each county's culture industry in a more comprehensive, efficient and healthy way.

Presently, the domestic researches on the development level of culture industry are abundant. For example, Zhang Qiang and Long Lin(2005) did a preliminary attempt to establish a index system for Yunnan Province's national culture industry consulting the constructing method of the tertiary industry and culture industry in statistical index[2].Xu Ping(2007) chose nineteen indexes from China Statistical Yearbook on Culture and Related Industries2004,China Statistical Yearbook-2004,China Statistical Yearbook on Population-2004 to construct a comprehensive index system in order to evaluate the development level and find out the development characteristic of the eastern area, the middle area and the western area in China by applying the technique of factor analysis[3]. Xue Hong(2010)created a pair of evaluation index for Nantong County in order to evaluate the development level of culture industry meanwhile the author used objective method--entropy value method to reflect the comprehensive situation of culture industry[1].Zhou Jin and Wen wen(2012) chose indexes from different provinces' Statistical Yearbook to create a pair of evaluation index system to analyse and evaluate the 
developmental state of culture industry of thirty one provinces in China[4].Zhang Huili and Wang Chengjun(2013)consulted the idea proposed by UNESO in India and Port's diamond theory ,then established a comprehensive evaluation system index to evaluate the developmental state of culture industry in Shanxi Province by applying the technique of factor analysis[5]. LiMingyu(2016) constructed the index system of the comprehensive level evaluation of the cultural and creative industries and uses principal component analysis to evaluate the comprehensive level of the cultural and creative industries within each district and county of Beijing. On this basis, the 16 counties are divided into five areas, including traditional cultural area, cultural fusion of science and technology area, the comprehensive improved area of the cultural and creative industries, the potential mining area of the cultural and creative industries, and the development area of ecological culture. So each district in Beijing may make full use of the resource advantages and industry characteristics, and promote the development level of the cultural and creative industries[6].

To sum up in conclusion, we find that most of above papers pay mainly attention to evaluating the developmental state of cultural industry at provincial scale ,but rarely papers conduct research on evaluating the developmental state of cultural industry by county. At the same time, the author find that after carefully searching references barely papers specialize in doing researches on the developmental state of culture industry by county in Guizhou Province. Given these, this paper attempts to construct comprehensive evaluation system index to evaluate the developmental state of culture industry by county specializing in Guizhou Province. This index system can be divide into mainly three parts ,respectively, industrial productivity, industrial influence, industrial driver, and altogether include twenty three indexes and we apply principal component analysis to process data and appraise the developmental state of culture industry of eighty eight counties in Guizhou Province.

\section{THE CONSTRUCTION OF EVALUATION INDEX SYSTEM}

\section{A. Theoretical Base of Evaluation Index System}

The theoretical framework is based on the Evaluation of National Cultural Industry in the Asia-Pacific Region proposed by the UNESCO in Jodhpur forum, in order to fit our country's national conditions, the authors modify some of the indexes from two aspects, respectively, industrial input and industrial output[7].

The theoretical base on how to choose index is Michael Porter diamond Model which can be used to analyse different countries' industrial competitiveness. Culture industry has general characters of industry, therefore, it is suitable to refer to the components of Diamond Model in order to analyse culture industry competitiveness[7].

Based on the above theoretical model and realistic conditions of culture industry in Guizhou Province, we put forward an comprehensive evaluation index system to evaluate each county's developmental status of culture industry in Guizhou Province. We divide our index system into three levels ,the first level includes three aspects, respectively, industrial productivity, industrial influence, industrial driver; the second level includes eight aspects, respectively, culture resource, culture capital, human resource, economic influence, social influence, market environment, public environment, innovation environment; the third level includes twenty-four aspect, we will give clear details about these aspect in the following table.

\section{B. The Principle of How to Choose Index}

The template is used to format your paper and style the text. All margins, column widths, line spaces, and text fonts are prescribed; please do not alter them. You may note peculiarities. For example, the head margin in this template measures proportionately more than is customary. This measurement and others are deliberate, using specifications that anticipate your paper as one part of the entire proceedings, and not as an independent document. Please do not revise any of the current designations.

This paper follow the following principle to choose index,namely,scientificalness, diversity,operability,systemati cness.Scientificalness means the guiding thinking should be reasonable and the indexes we choose must be based the facts; diversity means any two indexes we choose reflect different content ;operability indicates the data related to the indexes we choose is available; systematicness indicates the indexes we choose are able to reflect almost every aspects that have some extent impact on the development of culture industry.

\section{The Evaluation Index System of County in Guizhou Province}

According to the theoretical base and principle, we choose twenty-three third level indexes to reflect and evaluate the developmental state of county cultural industry in Guizhou Province, the complete index system is in table 1.

TABLE I. THE EVALUATION INDEX SYSTEM OF COUNTY IN GUIZHOU PROVINCE

\begin{tabular}{|c|c|c|}
\hline The first level & $\begin{array}{c}\text { The second } \\
\text { level }\end{array}$ & The third level \\
\hline \multirow{3}{*}{$\begin{array}{l}\text { industrial } \\
\text { productivity }\end{array}$} & culture resource & $\begin{array}{l}\text { the number of Intangible Cultural } \\
\text { Heritage( } X 1 \text { ) the proportion of } \\
\text { population of Minority in total } \\
\text { population( } X 2 \text { )、 the number of } \\
\text { cultural enterprise }(X 3)\end{array}$ \\
\hline & culture capital & $\begin{array}{l}\text { fixed assets investment per capita in } \\
\text { cultural industry ( } X 4 \text { ) 、 social } \\
\text { fixed assets investment in cultural } \\
\text { industry }(X 5)\end{array}$ \\
\hline & $\begin{array}{l}\text { Human } \\
\text { resource }\end{array}$ & $\begin{array}{l}\text { the employed population in cultural } \\
\text { industry }\left(\begin{array}{ll}X & 6\end{array}\right)\end{array}$ \\
\hline \multirow{2}{*}{$\begin{array}{l}\text { industrial } \\
\text { influence }\end{array}$} & $\begin{array}{l}\text { economic } \\
\text { influence }\end{array}$ & $\begin{array}{c}\text { total output of cultural } \\
\text { industry }(X 7) \text {, per capita income } \\
\text { of cultural industry ( } X 8)\end{array}$ \\
\hline & social influence & $\begin{array}{l}\text { the number of large-scale cultural } \\
\text { activity }(X 9) \text {, the proportion of } \\
\text { broadcasting time of self-made }\end{array}$ \\
\hline
\end{tabular}




\begin{tabular}{|c|c|c|}
\hline & & $\begin{array}{l}\text { activities in total broadcasting time } \\
\text { ( } X 10) \text {, cultural } \\
\text { atmosphere }(X 11) \text {, cultural } \\
\text { figure }(X 12) \text {, cultural } \\
\text { tolerance }(X 13)\end{array}$ \\
\hline \multirow{3}{*}{ industrial driver } & $\begin{array}{c}\text { market } \\
\text { environment }\end{array}$ & $\begin{array}{l}\text { total retail sales of consumer } \\
\text { goods }(X 14) \text {, per capita } \\
\text { disposable income of urban } \\
\text { residents }(X 15) \text {, the number of } \\
\text { tourist reception annually }(X 16) \text {, } \\
\text { the number of internet } \\
\text { user } X 17) \text {, balance of RMB } \\
\text { loans of financial } \\
\text { institutions }(X 18)\end{array}$ \\
\hline & $\begin{array}{c}\text { public } \\
\text { environment }\end{array}$ & $\begin{array}{l}\text { the proportion of cultural budget in } \\
\text { fiscal } \\
\text { budget }(X 19) \text {, general public bu } \\
\text { dget }(X 20)\end{array}$ \\
\hline & $\begin{array}{l}\text { innovation } \\
\text { environment }\end{array}$ & $\begin{array}{l}\mathrm{R} \& \mathrm{D} \text { expenditure for Humanities } \\
\text { and Social Sciences( } X 21) 、 \mathrm{R} \& \\
\mathrm{D} \text { personnel of Humanities and } \\
\text { Social Sciences( } X 22 \text { )、 } \mathrm{R} \& \mathrm{D} \\
\text { activities in the Humanities and } \\
\text { Social Sciences(Published scientific } \\
\text { and technological papers)( } X 23)\end{array}$ \\
\hline
\end{tabular}

According to the above table, we know the first level includes three indexes, the first index industrial productivity indicates each county's endowment; the second index industrial influence can be divide into two parts ,one part is social influence, this part reflects each county's cultural atmosphere and environment; the other is economic influence, this part indicates the economic income result from culture industry; the third index industrial driver indicates the external contributors that foster the development of culture industry. For convenience, we use $X 1 \ldots \ldots{ }^{X 23}$ to indicate each third index.

\section{EMPIRICAL ANALYSIS}

\section{A. The Source of Data}

The data has three sources the first source is field research and investigation; the second source is Guizhou Province Statistical Yearbook-2014; the third part comes from expert scoring.

\section{B. Empirical Result and Analysis}

This paper uses principal component analysis to evaluate the developmental state of cultural industry and we apply SPSS19.0 to do the analysis.

We divide the analysis process into the following steps:

Step one: Before analyse the data with principal component analysis, we will check the efficiency of these data to make sure whether these data can be analysed with principal component analysis. The test result can be found in Table 2:
TABLE II. KMO AND BARTLETT'S TEST OF SPHERICITY

\begin{tabular}{|c|c|}
\hline $\begin{array}{c}\text { Kaiser-Meyer-Olkin Measure of } \\
\text { Sampling Adequacy }\end{array}$ & 0.792 \\
\hline $\begin{array}{c}\text { Bartlett's Test of Sphericity } \\
\text { Approx.Chi-Square }\end{array}$ & 3282.039 \\
\hline df & 253 \\
\hline Sig. & 0.000 \\
\hline
\end{tabular}

According to above table, we find that KMO is 0.792 ( $>0.5$ ), Sig is $0.000(\langle 0.05)$.So the data we collect is suitable for us to analyse them with principal component analysis.

Step two: we use SPSS19.0 to get communalities. From the table, we can find most of the indexes' extraction is more than 0.8 , which indicates the indexes we choose have strong explanatory power.

Step three: in this step, our main aim is to find the components whose eigenvalue is not less than one, because only if the component's corresponding eigenvalue is not less than one, then this component can have strongly enough comprehensive explanatory power so that to represent the combination of above indexes. In view of above reasons, we set eigenvalues not less than one in SPSS19.0,so we can get following table.

TABLE III. TOTAL VARIANCE EXPLAINED

\begin{tabular}{|c|c|c|c|c|c|c|}
\hline \multicolumn{7}{|c|}{ Total variance explained } \\
\hline \multirow{2}{*}{$\begin{array}{c}\text { comp } \\
\text { onent }\end{array}$} & Total & $\begin{array}{c}\text { \% of } \\
\text { variance }\end{array}$ & $\begin{array}{c}\text { cumulativ } \\
\text { e } \%\end{array}$ & total & $\begin{array}{c}\text { Of } \\
\text { variance \% } \%\end{array}$ & $\begin{array}{c}\text { cumulativ } \\
\text { e \% }\end{array}$ \\
\cline { 2 - 7 } & 10.578 & 45.989 & 45.989 & 10.578 & 45.989 & 45.989 \\
\hline 1 & 2.523 & 10.968 & 56.957 & 2.523 & 10.968 & 56.957 \\
\hline 2 & 1.939 & 8.431 & 65.388 & 1.939 & 8.431 & 65.388 \\
\hline 3 & 1.356 & 5.896 & 71.284 & 1.356 & 5.896 & 71.284 \\
\hline 5 & 1.214 & 5.279 & 76.563 & 1.214 & 5.279 & 76.563 \\
\hline 6 & 1.070 & 4.650 & 81.214 & 1.070 & 4.650 & 81.214 \\
\hline & Extraction method: principal component analysis & \\
\hline
\end{tabular}

Step four: the key function of this step is to help us to know the extracting share of each component in every index. According to the component matrix, we can calculate each expression formula of principal component, for notation simplicity, we use a series letters to be the indicator of each principal component, namely, $Y 1 、 Y 2 、 Y 3 、 Y 4$ 、 $Y 5 、 Y 6 . Y 1$ represents the first component, $Y 2$ represents the second component, and the like. In order to attain the coefficient of each index of expression formula of principal component, we use each index's extracting share showed in the component matrix over each principal component's corresponding eigenvalue, then we can get each expression formula of principal component. 
$Y 1=-0.084 X 1-0.175 X 2+0.119 X 3+0.164 X 4+0.253 X 5+0.159 X 6+0.276 X 7$ $+0.246 X 8+0.038 X 9+0.045 X 10+0.038 X 11+0.221 X 12-0.174 X 13+0.280 X 14+$ $0.261 X 15+0.215 X 16+0.256 X 17+0.271 X 18-0.041 X 19+0.257 X 20+0.257 X 21$ $+0.264 \times 22+0.275 \times 23$

$$
\begin{gathered}
Y 2=0.128 X 1+\ldots \ldots+0.199 X 23 \\
Y 3=0.156 X 1+\ldots \ldots+0.117 X 23 \\
Y 4=0.314 X 1+\ldots \ldots-0.087 X 23 \\
Y 5=0.229 X 1+\ldots . .-0.080 X 23 \\
Y 6=0.669 X 1+\ldots \ldots-0.076 X 23
\end{gathered}
$$

In accordance with above equation, we can calculate each principal component's scores, the detailed result can be found in the following table, namely, table 6 .

We use $\mathrm{Z}$ to indicate each county's comprehensive scores. According to table 4,we can get expression formula of $\mathrm{Z}$, the coefficient of $\mathrm{Y}$ is weighted mean of eigenvalue.

$$
Z=0.566 Y 1+0.135 Y 2+0.104 Y 3+0.073 Y 4+0.065 Y 5+0.057 Y 6
$$

According to equation 7, we can find that the coefficient of the principal component decrease in sequence. The first principal component has the biggest influence on $\mathrm{Z}$, because the coefficient of the first principal component account for $56 \%$ of the total,, the better part of $\mathrm{Z}$ comes from the first principal component. Compared with the first, the impact from the second principal component on $\mathrm{Z}$ apparently decrease, while it is a bit higher than the other principal component. The remaining principal component has similar situation, we don't repeat them here.

According to equation (1)(2)(3)(4)(5)(6)(7), we can get each principal component's score and each county's

\begin{tabular}{|c|c|c|c|c|c|c|c|c|}
\hline $\begin{array}{c}\text { coun } \\
\text { ty }\end{array}$ & $\begin{array}{l}\text { Yunyan } \\
\text { County }\end{array}$ & $\begin{array}{c}\text { Nanming } \\
\text { County }\end{array}$ & $\begin{array}{l}\text { Huaxi } \\
\text { County }\end{array}$ & $\begin{array}{l}\text { Xingyi } \\
\text { County }\end{array}$ & $\begin{array}{c}\text { Kaili } \\
\text { County }\end{array}$ & \begin{tabular}{|c|} 
Guanshan \\
hu \\
County
\end{tabular} & $\begin{array}{c}\text { Honghuag } \\
\text { ang } \\
\text { County }\end{array}$ & $\begin{array}{c}\text { Qingzhen } \\
\text { County }\end{array}$ \\
\hline Z & 9.464322 & 8.286893 & $\begin{array}{c}5.0387 \\
36\end{array}$ & $\begin{array}{c}2.8611 \\
93\end{array}$ & $\begin{array}{c}2.6694 \\
09\end{array}$ & 2.654573 & 2.580816 & 2.185733 \\
\hline $\mathrm{R}$ & 1 & 2 & 3 & 4 & 5 & 6 & 7 & 8 \\
\hline $\begin{array}{c}\text { coun } \\
\text { ty }\end{array}$ & \begin{tabular}{|c|} 
Zhongsha \\
ng \\
County \\
\end{tabular} & $\begin{array}{l}\text { Baiyun } \\
\text { County }\end{array}$ & $\begin{array}{c}\text { Pan } \\
\text { County }\end{array}$ & \begin{tabular}{|c|} 
Kaiyan \\
$\mathrm{g}$ \\
County
\end{tabular} & \begin{tabular}{|c|} 
Renhu \\
ai \\
County \\
\end{tabular} & $\begin{array}{c}\text { Huichuan } \\
\text { County }\end{array}$ & $\begin{array}{l}\text { Wudang } \\
\text { County }\end{array}$ & $\begin{array}{l}\text { Xixiu } \\
\text { County }\end{array}$ \\
\hline Z & 2.081878 & 2.070734 & $\begin{array}{c}1.8758 \\
56\end{array}$ & $\begin{array}{c}1.7008 \\
21 \\
\end{array}$ & $\begin{array}{c}1.6403 \\
77\end{array}$ & 1.573479 & 1.497998 & 1.336979 \\
\hline $\mathrm{R}$ & 9 & 10 & 11 & 12 & 13 & 14 & 10 & 16 \\
\hline $\begin{array}{c}\text { coun } \\
\text { ty }\end{array}$ & $\begin{array}{l}\text { Zunyi } \\
\text { county }\end{array}$ & \begin{tabular}{|c|} 
Qixinggu \\
an \\
County
\end{tabular} & \begin{tabular}{|c|} 
Xiuwe \\
$n$ \\
County
\end{tabular} & $\begin{array}{l}\text { Xifeng } \\
\text { County }\end{array}$ & $\begin{array}{l}\text { Bijiang } \\
\text { County }\end{array}$ & $\begin{array}{l}\text { Shuichen } \\
\text { g County }\end{array}$ & $\begin{array}{l}\text { Jingsha } \\
\text { County }\end{array}$ & $\begin{array}{l}\text { Duyun } \\
\text { County }\end{array}$ \\
\hline Z & 1.227441 & 1.08079 & $\begin{array}{c}0.8852 \\
85\end{array}$ & $\begin{array}{c}0.6262 \\
44\end{array}$ & $\begin{array}{c}0.4614 \\
95\end{array}$ & 0.352229 & 0.255817 & 0.216373 \\
\hline
\end{tabular}
comprehensive score, then we give each county a rank based

\begin{tabular}{|c|c|c|c|c|c|c|c|c|}
\hline $\mathrm{R}$ & 17 & 18 & 19 & 20 & 21 & 22 & 23 & 24 \\
\hline $\begin{array}{c}\text { coun } \\
\text { ty }\end{array}$ & $\begin{array}{l}\text { Meitan } \\
\text { County }\end{array}$ & $\begin{array}{l}\text { Tongzhi } \\
\text { County }\end{array}$ & $\begin{array}{l}\text { Zhijin } \\
\text { County }\end{array}$ & $\begin{array}{c}\text { Fuquan } \\
\text { Count }\end{array}$ & $\begin{array}{l}\text { Yuqing } \\
\text { County }\end{array}$ & $\begin{array}{l}\text { Qianxi } \\
\text { County }\end{array}$ & $\begin{array}{l}\text { Jinping } \\
\text { County }\end{array}$ & $\begin{array}{l}\text { Chishui } \\
\text { County }\end{array}$ \\
\hline Z & -0.03963 & -0.11331 & $\begin{array}{c}- \\
0.1267 \\
4\end{array}$ & $\begin{array}{c}- \\
0.1834 \\
7\end{array}$ & $\begin{array}{c}- \\
0.2177 \\
3\end{array}$ & -0.26364 & -0.28932 & -0.28976 \\
\hline $\mathrm{R}$ & 25 & 26 & 27 & 28 & 29 & 30 & 31 & 32 \\
\hline $\begin{array}{c}\text { coun } \\
\text { ty }\end{array}$ & $\begin{array}{l}\text { Dafang } \\
\text { County }\end{array}$ & $\begin{array}{l}\text { Pingba } \\
\text { County }\end{array}$ & $\begin{array}{l}\text { Wenan } \\
\text { County }\end{array}$ & $\begin{array}{c}\text { Zhenyu } \\
\text { an } \\
\text { County }\end{array}$ & $\begin{array}{l}\text { Puding } \\
\text { County }\end{array}$ & $\begin{array}{c}\text { Zhenfeng } \\
\text { County }\end{array}$ & $\begin{array}{l}\text { Liuzhi } \\
\text { County }\end{array}$ & $\begin{array}{l}\text { Xishui } \\
\text { County }\end{array}$ \\
\hline Z & -0.29265 & -0.29268 & $\begin{array}{c}- \\
0.3951 \\
9\end{array}$ & $\begin{array}{c}- \\
0.4003 \\
5\end{array}$ & $\begin{array}{c}- \\
0.4421 \\
3\end{array}$ & -0.51136 & -0.5115 & -0.51824 \\
\hline $\mathrm{R}$ & 33 & 34 & 35 & 36 & 37 & 38 & 39 & 40 \\
\hline $\begin{array}{c}\text { coun } \\
\text { ty }\end{array}$ & $\begin{array}{l}\text { Weining } \\
\text { County }\end{array}$ & $\begin{array}{l}\text { Yanhe } \\
\text { County }\end{array}$ & $\begin{array}{l}\text { Longli } \\
\text { County }\end{array}$ & $\begin{array}{c}\text { Suiyan } \\
\text { g } \\
\text { County }\end{array}$ & $\begin{array}{c}\text { Nayon } \\
\mathrm{g} \\
\text { County }\end{array}$ & $\begin{array}{c}\text { SongTao } \\
\text { County }\end{array}$ & $\begin{array}{l}\text { Sinan } \\
\text { County }\end{array}$ & $\begin{array}{l}\text { Guiding } \\
\text { County }\end{array}$ \\
\hline Z & -0.54376 & -0.56756 & $\begin{array}{c}- \\
0.6242 \\
6\end{array}$ & $\begin{array}{c}- \\
0.6488 \\
3\end{array}$ & $\begin{array}{c}- \\
0.6599 \\
7\end{array}$ & -0.6911 & -0.7302 & -0.77547 \\
\hline $\mathrm{R}$ & 41 & 42 & 43 & 44 & 45 & 46 & 47 & 48 \\
\hline $\begin{array}{c}\text { coun } \\
\text { ty }\end{array}$ & $\begin{array}{l}\text { Congjian } \\
\text { g County }\end{array}$ & $\begin{array}{c}\text { Huangpi } \\
\text { ng } \\
\text { County }\end{array}$ & $\begin{array}{l}\text { Yuping } \\
\text { County }\end{array}$ & $\begin{array}{c}\text { Guanli } \\
\text { ng } \\
\text { County }\end{array}$ & $\begin{array}{c}\text { DuSha } \\
n \\
\text { County }\end{array}$ & $\begin{array}{c}\text { Zhenning } \\
\text { County }\end{array}$ & Leishan & $\begin{array}{l}\text { Jiangko } \\
\text { County }\end{array}$ \\
\hline Z & -0.77675 & -0.79278 & $\begin{array}{c}- \\
0.8228 \\
6\end{array}$ & $\begin{array}{c}- \\
0.8626 \\
8 \\
\end{array}$ & $\begin{array}{c}- \\
0.8629 \\
6 \\
\end{array}$ & -0.89427 & -0.90947 & -0.91168 \\
\hline $\mathrm{R}$ & 49 & 50 & 51 & 52 & 53 & 54 & 55 & 56 \\
\hline $\begin{array}{c}\text { coun } \\
\text { ty }\end{array}$ & $\begin{array}{c}\text { Xingren } \\
\text { County }\end{array}$ & $\begin{array}{l}\text { Fenggan } \\
\text { g County }\end{array}$ & $\begin{array}{l}\text { Huishu } \\
\text { i } \\
\text { County }\end{array}$ & $\begin{array}{l}\text { Anlong } \\
\text { County }\end{array}$ & $\begin{array}{c}\text { Hezha } \\
\text { ng } \\
\text { County }\end{array}$ & $\begin{array}{l}\text { Cenggon } \\
\text { g County }\end{array}$ & $\begin{array}{l}\text { Majiang } \\
\text { County }\end{array}$ & $\begin{array}{c}\text { Changshun } \\
\text { County }\end{array}$ \\
\hline Z & -0.91501 & -0.91881 & $\begin{array}{c}- \\
0.9738 \\
3\end{array}$ & $\begin{array}{c}- \\
0.9789 \\
6\end{array}$ & $\begin{array}{c}- \\
0.9813 \\
3\end{array}$ & -0.99508 & -1.01061 & -1.02291 \\
\hline $\mathrm{R}$ & 57 & 58 & 59 & 60 & 61 & 62 & 63 & 64 \\
\hline $\begin{array}{c}\text { coun } \\
\text { ty }\end{array}$ & $\begin{array}{c}\text { Pingtang } \\
\text { County }\end{array}$ & $\begin{array}{l}\text { Liping } \\
\text { County }\end{array}$ & $\begin{array}{c}\text { Yinjian } \\
\mathrm{g} \\
\text { County }\end{array}$ & $\begin{array}{c}\text { Tianzh } \\
\text { u } \\
\text { County }\end{array}$ & \begin{tabular}{|c|} 
Puan \\
County
\end{tabular} & $\begin{array}{c}\text { Danzhai } \\
\text { County }\end{array}$ & $\begin{array}{l}\text { Dejiang } \\
\text { County }\end{array}$ & $\begin{array}{l}\text { Shibing } \\
\text { County }\end{array}$ \\
\hline Z & -1.02589 & -1.03446 & $\begin{array}{c}- \\
1.0541 \\
6\end{array}$ & $\begin{array}{c}- \\
1.0773 \\
9\end{array}$ & $\begin{array}{c}- \\
1.1163 \\
9\end{array}$ & -1.12146 & -1.1443 & -1.14555 \\
\hline $\mathrm{R}$ & 65 & 66 & 67 & 68 & 69 & 70 & 71 & 72 \\
\hline $\begin{array}{c}\text { coun } \\
\text { ty }\end{array}$ & $\begin{array}{c}\text { Libo } \\
\text { County } \\
\end{array}$ & $\begin{array}{l}\text { Sansui } \\
\text { County }\end{array}$ & $\begin{array}{c}\text { Shiqia } \\
n \\
\text { County }\end{array}$ & $\begin{array}{c}\text { Zhenga } \\
n \\
\text { County }\end{array}$ & $\begin{array}{c}\text { Sandu } \\
\text { County }\end{array}$ & $\begin{array}{l}\text { Ziyun } \\
\text { County }\end{array}$ & $\begin{array}{c}\text { Wangmo } \\
\text { County }\end{array}$ & $\begin{array}{c}\text { Qinglong } \\
\text { County }\end{array}$ \\
\hline Z & -1.14563 & -1.17778 & $\begin{array}{c}- \\
1.1941 \\
1\end{array}$ & $\begin{array}{c}- \\
1.1954 \\
3\end{array}$ & $\begin{array}{c}- \\
1.2671 \\
6\end{array}$ & -1.27901 & -1.28615 & -1.28783 \\
\hline $\mathrm{R}$ & 73 & 74 & 75 & 76 & 77 & 78 & 79 & 80 \\
\hline $\begin{array}{c}\text { coun } \\
\text { ty }\end{array}$ & $\begin{array}{l}\text { Taijiang } \\
\text { County }\end{array}$ & $\begin{array}{l}\text { Rongjian } \\
\text { g County }\end{array}$ & $\begin{array}{l}\text { Daozh } \\
\text { en } \\
\text { County }\end{array}$ & \begin{tabular}{|l|} 
Jianhe \\
County
\end{tabular} & $\begin{array}{c}\text { Wansh } \\
\text { an } \\
\text { County }\end{array}$ & $\begin{array}{c}\text { Wuchuan } \\
\text { County }\end{array}$ & $\begin{array}{l}\text { Luodian } \\
\text { County }\end{array}$ & $\begin{array}{l}\text { Cexiang } \\
\text { County }\end{array}$ \\
\hline Z & -1.34925 & -1.35643 & $\begin{array}{c}- \\
1.3895 \\
4\end{array}$ & $\begin{array}{c}- \\
1.3960 \\
6\end{array}$ & $\begin{array}{c}- \\
1.4094 \\
3\end{array}$ & -1.42641 & -1.45873 & -1.50916 \\
\hline $\mathrm{R}$ & 81 & 82 & 83 & 84 & 85 & 86 & 87 & 88 \\
\hline
\end{tabular}
on its comprehensive score in order to reflect its developmental state of cultural industry. The detailed data is in the following table 6 .

\section{TABLE IV. RANK(R) AND SCORE}

According to table 6,we divide these counties' developmental state of cultural industry into four parts based on their comprehensive scores. The first category is ' $\operatorname{good}(Z \geq 1)$; the second category is 'medium' $(0 \leq Z<1)$, the third category is 'below medium' $(-1 \leq Z<0)$; the fourth 
category is 'bad' $(Z<-1)$.According to above classification, we can obtain the following table 5 .

TABLE V. THE CATEGORY OF DEVELOPMENT STATE

\begin{tabular}{|c|c|c|c|c|}
\hline & Good & Medium & $\begin{array}{c}\text { Below } \\
\text { medium }\end{array}$ & Bad \\
\hline number & 18 & 6 & 38 & 26 \\
\hline
\end{tabular}

According to above table 7,we find the number of county whose developmental state of cultural industry is above medium only 24,that is to say, the remaining 64 are below the medium ,therefore, the developmental state of cultural industry in Guizhou Province is under-developped on the whole.

Let us do an elaborate and comprehensive exploration in above four categories. the comprehensive score ranking above the medium among the counties, which ranked in the top 24 counties, the scores of the first principal component are also in the top 24 and most counties' scores in the first principal component are more than 2 points. In addition, the comprehensive scores are ranked in the bottom 10 , the first principal component scores are rightly ranked in the bottom 10.In view of the above facts, the first principal component has a great influence on the county cultural industry development level. According to table five, we find the following indexes have greatly positive on the first component ,respectively, $X 5, X 7, X 8, X 14, X 15, X 17, X 18, X 20, X 21$ $, x 22, x 23$.According to the index system we establish, we find culture capital, economic influence, market environment, public environment, innovation environment have great influence on cultural industry, and in the above five aspects the public environment and innovation environment are particularly important. Therefore the relative backward counties in the area of cultural industry should focus on the promotion of cultural capital, economic impact, market environment, public environment, development and innovation environment so that to promote the development of cultural industry in a more more efficient way.

Apart from that the first principal component has obvious impact on the comprehensive scores, the other principal also has contribution to the comprehensive scores. Therefore it is necessary for us to explore what's the factors that influence these principal component. According to table five, we find the second principal component is positively and closely related to $x_{6}, x^{x}$ and $x 11$;the third principal component is positively and closely related to $x 2$ and $x 13$; the fourth principal component is positively and closely related to $X 3$ and $X 16$; the fifth principal component is positively and closely related to $X 10$ and $X 19$;the sixth principal component is positively and closely related to $X 1$.

Further analysis of table six, we find the development level of cultural industry is various from counties to counties. Taking the top 1 Yunyan County as an example, the score in the first and the second principal components is much higher than other districts and its comprehensive score is more 11 points than the lowest county Cexiang County ,in addition, its comprehensive score is 50 times of Duyun County whose rank is the twenty-forth. But, its point in the fifth and sixth principal component is higher less than the above two county. Therefore, we can see that Guizhou Province, in addition that the level of development of cultural industry overall is relatively backward, the level of development of cultural industry is in general of poor balance.

\section{CONCLUSION}

In order to learn of the development state of cultural industry in Guizhou Province, this paper applies principal component analysis with the help of SPSS 19.0.Firstly,we established index system of the development level of culture industry. Then, we used SPSS 19.0 to standardize the statistics, followingly, we did principal component analysis with the standardized the statistics and we got six principal components. Finally, we applied SPSS 19.0 to analyse the result and got the scores of every principal component, then we used each principal component's eigenvalue as weight to calcuate each county's weighted scores and got the rank based on the weighted scores. The main contribution of this paper is to establish a comprehensive index system, and find out the advantages and disadvantages of the county cultural industry in Guizhou Province. The shortages of this article is on the one hand the data we collect is not enough, on the other hand the index system we establish is not comprehensive. Therefore, the next work is to enhance the accuracy of explanation from every aspect.

\section{ACKNOWLEDGMENT}

This work was financially supported by National Natural Science Foundation of China (71263011).

\section{REFERENCES}

[1] Xue Hong, "Research on The Index System of Cultural Industry --a Case Study of Cultural Industry in Nantong City,Jiangsu Province”China Statistics, vol. 12, 2010, pp. 45-46

[2] Zhang Qiang and Long Lin , "The Construction of Evaluation Index System of National Cultural Industry” Inquiry into Economic Problems, vol. 6, 2005,pp. 50-53

[3] Xu Ping, "The Development Level and Characteristics of Regional Cultural Industry in China” Statistics and Decision, vol.1 2007, pp. 75-76

[4] Zhou Jin and Wen Wen, "Evaluation of Chinese Cultural Industry Development Based on Factor Analysis”,East China Economic Management, vol. 2, 2012, pp. 46-50

[5] Zhang Huili and Wang Chengjun ,"Comprehensive Evaluation of City Culture Industry Development Level”Science and Technology Management research, vol. 19, 2013, pp. 221-224

[6] Li Mingyu, “The Study of Developmeng Level and Functional Partition of Cultural and Creative Industries in Beijing Based on Principal Component Analysis”Ecological Economy, Vol. 32, March 2016, pp. 127-129

[7] Peng Yi,China Urban Cultural Industry Development Evaluation Index System Research,2rd,Beijing:People's University Press,pp. 5053 